\title{
Utilização das técnicas de imuno-histoquímica e reação em cadeia da polimerase (PCR) no diagnóstico, na avaliação de fatores prognósticos e no direcionamento do tratamento do câncer de pulmão
}

\section{Use of immunohistochemistry techniques and polymerase chain reaction (PCR) in diagnosis, in the evaluation of prognostic factors and in the direction of treatment of lung cancer}

Carlos Eduardo Lopes Soares ${ }^{1}$. Pâmela Maria Martins da Silva Xavier ${ }^{1}$. Ana Cláudia da Silva Mendes de Oliveira $^{2}$. Gunter Gerson ${ }^{1}$.

1 Universidade Federal do Ceará (UFC), Fortaleza, Ceará, Brasil. 2 Hospital de Messejana Dr. Carlos Alberto Studart Gomes, Fortaleza, Ceará, Brasil.

\section{RESUMO}

Introdução: O câncer de pulmão é uma neoplasia de alta incidência e mortalidade. A identificação da imunoexpressão de PDL1 tem possibilitado novos tratamentos com anticorpos monoclonais e a técnica de PCR para pesquisa de alterações do receptor do fator de crescimento epidérmico (EGFR) permite indicar o tratamento com inibidores de tirosina quinase. Objetivo: avaliar o perfil imuno-histoquímico e as alterações do EGFR em uma série de amostras de neoplasia pulmonar. Metodologia: Estudo observacional retrospectivo de uma série de 29 amostras de neoplasia pulmonar do serviço de biópsia do departamento de patologia da Universidade Federal do Ceará, entre agosto de 2016 e março de 2020. Resultados: Houve maior prevalência em mulheres (66\%), com idade média de 66 anos. Tumores primários representaram a maioria dos casos (86\%). O tipo histológico predominante foi o adenocarcinoma (38\%), seguido do carcinoma de células escamosas (17\%), carcinoides (17\%), metástase (14\%) e outros tumores (14\%). Entre os adenocarcinomas, o subtipo acinar foi mais frequente (45\%). Entre os tumores secundários, metade eram metástase de neoplasia hepática. Expressão de PDL-1 e alteração de EGFR foram avaliadas em 16 casos, sendo observado em $12 \%$ e 25\% dos casos, respectivamente. Conclusão: Este estudo não mostrou relação entre os casos que apresentaram imunoexpressão de PDL-1 e alterações de EGFR.

Palavras-chave: Neoplasias pulmonares. Imuno-histoquímica. Reação em cadeia da polimerase.

\section{ABSTRACT}

Introduction: Lung cancer is a cancer of high incidence and mortality. The identification of PD-L1 immunoexpression has enabled new treatments with monoclonal antibodies, and the PCR technique for researching Epidermal Growth Factor Receptor (EGFR) changes allows indicating treatment with tyrosine kinase inhibitors. Objective: to evaluate the immunohistochemical profile and EGFR changes in a series of lung cancer samples. Methodology: Retrospective observational study of a series of 29 lung cancer samples from the biopsy service of the department of pathology at the Universidade Federal do Ceará, between August 2016 and March 2020. Results: There was a higher prevalence in women (66\%), with an average age of 66 years. Primary tumors represented the majority of cases (86\%). The predominant histological type was adenocarcinoma (38\%), followed by squamous cell carcinoma (17\%), carcinoids (17\%), metastasis (14\%) and other tumors (14\%). Among adenocarcinomas, the acinar subtype was more frequent (45\%). Among secondary tumors, half were metastatic to liver cancer. PDL-1 expression and EGFR alteration were evaluated in 16 cases, with $12 \%$ and $25 \%$ of cases observed, respectively. Conclusion: This study showed no relationship between the cases that showed PDL-1 immunoexpression and EGFR changes.

Keywords: Lung neoplasms. Immunohistochemistry. Polymerase chain reaction.

Autor correspondente: Carlos Eduardo Lopes Soares, Rua 09, número 249, Vila das Flores, Pacatuba, Ceará. CEP: 61814-456. Telefone: +55 85 98856-7392. E-mail: eduardolopes.ti@gmail.com

Conflito de interesses: Não há qualquer conflito de interesses por parte de qualquer um dos autores.

Recebido em: 25 Nov 2020; Revisado em: 18 Set 2021; Aceito em: 21 Set 2021. 


\section{INTRODUÇÃO}

O câncer de pulmão é uma neoplasia com alta mortalidade, ocupando a primeira posição em todo o mundo, desde 1985, sendo um importante desafio para a oncologia. ${ }^{1,2}$ É o segundo mais comum em homens e o terceiro em mulheres. Segundo dados do Sistema de Informação de Mortalidade, houve 27.931 mortes em 2017. No Brasil, estimam-se 30.200 novos casos em $2020 .^{1}$

Os carcinomas pulmonares são classificados morfologicamente em carcinoma pulmonar de células não-pequenas (CNPC) e carcinoma pulmonar de células pequenas (CPPC). ${ }^{3}$ Aproximadamente $80 \%$ dos CNPCs são diagnosticados já na presença de doença localmente avançada ou metastática inoperável. Tumores carcinoides, linfomas e sarcomas são outros tipos histológicos de tumores pulmonares menos frequentes. ${ }^{4}$ As estratégias de tratamento atual envolvem a cirurgia, radioterapia, a quimioterapia e os agentes direcionados contra alvos específicos. ${ }^{5}$

A técnica de imuno-histoquímica ganha ampla utilização e fundamental importância no estudo destes tumores, definindo a histogênese e o subtipo tumoral, pesquisa de fatores prognósticos, direcionando tratamento e sobrevida desses pacientes. ${ }^{2,6}$ A identificação da imunoexpressão de PD-L1 tem possibilitado uma nova premissa de tratamento envolvendo o uso de anticorpos monoclonais, com respostas duráveis em alguns pacientes com doença avançada. ${ }^{7}$

A melhor compreensão da biologia molecular nas últimas décadas tem possibilitado a identificação de muitas proteínas associadas à carcinogênese dessa neoplasia, levando ao desenvolvimento de novos tratamentos promissores. A reação em cadeia da polimerase (PCR) para pesquisa de alterações no receptor do fator de crescimento epidérmico (EGFR) permite indicar o tratamento com inibidores de tirosina quinase, definindo o grupo de pacientes que pode ser tratado com eficácia por esta terapia direcionada específica. $^{2}$

O surgimento dos inibidores de tirosina quinase foi revolucionário no tratamento do câncer de pulmão. Essas drogas apresentam como possível alvo o oncogene receptor do fator de crescimento epidérmico (EGFR). O EGFR desempenha um papel importante na regulação das funções celulares e quando esse gene é superexpresso ou mutado nas células cancerígenas, influencia no tratamento, no prognóstico e na sobrevida. ${ }^{8}$ Mais recentemente, o desenvolvimento de fármacos bloqueadores do ligante de morte celular programada 1 (PD-L1) também resultou em grandes avanços no tratamento do câncer de pulmão. ${ }^{5,9} \mathrm{O}$ PD-L1 é o principal ligante da molécula de morte celular programada 1 (PD-1) e sua expressão na superfície de células tumorais regula e inibe a resposta imunológica no microambiente da neoplasia, sendo um mecanismo de escape tumoral do sistema imune. ${ }^{8}$

As células tumorais são reconhecidas pelo sistema imunológico, levando a uma resposta imune que causa a morte celular do câncer. Existe um mecanismo conhecido como evasão da resposta imune, onde o tumor expressa a molécula PD-L1, e assim as células tumorais bloqueiam as respostas imunes antitumorais, não sendo detectadas. ${ }^{10} \mathrm{~A}$ proteína de morte programada (PD1), que é uma proteína de checkpoint em células do sistema imunológico, as células $\mathrm{T}$, quando se liga ao seu ligante, a molécula PD-L1, limita as funções das células do sistema imune. O PD-L1 é expresso em grandes quantidades em alguns tumores.,11 Drogas que tem o alvo PD-1 ou PD-L1 tem se mostrado muito promissoras no tratamento, pois bloqueiam essa ligação e aumentam a respostas imune contra as células cancerígenas. ${ }^{4}$

\section{OBJETIVO}

Avaliar o diagnóstico, o perfil imuno-histoquímico e a técnica de PCR para pesquisa de alterações do EGFR em uma série de 29 amostras de tecido tumoral de pacientes com diagnóstico de neoplasia pulmonar.

\section{MATERIAIS E MÉTODOS}

Estudo transversal retrospectivo de uma série de 29 amostras de tecido tumoral de pacientes com diagnóstico de neoplasia pulmonar que tiveram suas amostras analisadas no serviço de biópsia do Departamento de Patologia e Medicina Legal da Universidade Federal do Ceará, entre agosto de 2016 e março de 2020. O estudo foi aprovado pelo Comitê de Ética e Pesquisa do Hospital Universitário Walter Cantídio.

As amostras dos casos selecionados foram revisadas, sendo realizadas releitura e análise de lâminas previamente confeccionadas por médico patologista com ampla experiência na área, a fim de avaliar e confirmar parâmetros patológicos: diagnóstico histopatológico e tipos morfológicos; perfil de expressão dos marcadores imuno-histoquímicos; além da revisão dos exames de PCR para pesquisa de alterações do EGFR.

Os aspectos clínicos foram obtidos das informações descritas em formulário padrão de solicitação de estudo anátomo-patológico enviadas ao setor de biópsia. Os parâmetros analisados foram apenas sexo e idade.

\section{Detalhamento do exame de imuno-histoquímica}

Operfil imuno-histoquímico foi avaliado após revisão ereleitura de lâminas com marcações específicas que foram realizadas nas amostras tumorais. Os referidos cortes foram submetidos aos métodos de imunocoloração, utilizando anticorpos específicos para diferenciação entre subtipos de neoplasias pulmonares, a depender da hipótese clínico-radiológica. Os principais marcadores imuno-histoquímicos utilizados foram: CK5/6; CK7; 34BE12; Cromogranina A; Sinaptofisina; Ki67; TTF-1 e p63.

Foram realizadas análises de forma descritiva, qualitativa, e os resultados apresentados em forma de gráficos e tabelas. 


\section{Detalhamento do exame de imuno-histoquímica para pesquisa de PD-L1}

A reação imuno-histoquímica da proteína PD-L1 foi realizada utilizando-se clone Dako 22C3 (DAKO Autostainer, AS480), seguindo o protocolo PD-L1 DAKO. O contraste feito com hematoxilina e controles negativos também foram avaliados.

Para interpretação dos resultados, foi avaliada apenas a expressão de PD-L1 em células neoplásicas. A marcação membranar de qualquer intensidade de PD-L1 foi definida como sendo positiva, a ausência de marcação como negativa.

\section{Detalhamento do exame de PCR para Pesquisa de alterações do EGFR}

A pesquisa de alterações do EGFR foi avaliada após revisão de laudos de exame de PCR. O exame foi realizado após seleção de material representativo de amostras dos tumores pulmonares, selecionando-se blocos com células neoplásicas fixados em formalina e incluídos em parafina que foram submetidos à desparafinização, à digestão e ao isolamento de DNA pelo DNA Simple Preparation Kit (Roche: 05985536190, Registro ANVISA: 10287410963), com pelo menos 10\% de tumor viável.

\section{Análise dos dados}

Os dados foram analisados através do software GraphPad Prism versão 6.0 para Windows, no qual as análises foram realizadas com intervalo de confiança de $95 \%$. A frequência absoluta e o percentual de cada variável foram expressos, sendo cruzados por meio dos testes exato de Fisher ou quiquadrado de Pearson. Um valor - P de $\leq 0,05$ foi considerado para indicar significância estatística.

\section{RESULTADOS}

Foram analisados um total de 29 casos. Destes, 19 (66\%) eram do sexo feminino. Os tumores primários de pulmão representaram 25 casos $(86 \%)$ e os tumores secundários 4 casos $(14 \%)$, sendo 2 casos de metástase de tumor primário do fígado, 1 da mama e 1 colorretal. O tipo histológico predominante, entre os tumores primários do pulmão, foi adenocarcinoma, no total de 11 casos (38\%), em seguida os casos de carcinoma de células escamosas (CEC) e tumor carcinoide, ambos com 5 casos cada (17\%), sendo 4 casos de metástase (14\%). Houve ainda 4 casos (14\%) de outros tumores menos frequentes, que foram carcinoma mucoepidermoide (CME) de baixo grau endobrônquico, hamartoma pleuropulmonar, hiperplasia adenomatosa atípica e linfoma de zona marginal extranodal. Todos os casos de metástase de tumor primário do fígado foram pós-transplante hepático (Tabela 1).

A maioria dos CNPCs eram de grau histológico moderadamente diferenciados, 12 casos (75\%), sendo 4 casos de grau pouco diferenciado (25\%). A expressão de PDL-1 foi estudada em 16 casos de CNPCs, sendo destes apenas 02 positivos (12\%) e
14 negativos (88\%). As alterações de EGFR foram estudadas também nos mesmos 16 casos, sendo 04 mutados (25\%) e 12 não-mutados (75\%) (Tabela 1$)$.

A idade média do total de pacientes analisados foi de $61,5 \pm$ 15,2 anos (30-85 anos), com mediana de 66 anos, sendo que 14 (48\%) tinham menos de 65 anos e 15 (52\%) mais de 65 anos. $\mathrm{Na}$ análise comparativa de idade ao diagnóstico e ao sexo, os homens tiveram idade média de 64,7 $\pm 13,2$ anos (45-85 anos) com mediana de 67 anos; as mulheres tiveram idade média de 59,8 $\pm 16,2$ anos (30-82 anos) com mediana de 64 anos.

Tabela 1. Informações gerais e dados epidemiológicos dos pacientes analisados.

\begin{tabular}{|c|c|c|}
\hline & $\mathrm{n}$ & $\%$ \\
\hline \multicolumn{3}{|l|}{ Sexo } \\
\hline Masculino & 10 & 34,4 \\
\hline Feminino & 19 & 65,6 \\
\hline \multicolumn{3}{|l|}{ Idade } \\
\hline$<65$ anos & 14 & 48,3 \\
\hline$>65$ anos & 15 & 51,7 \\
\hline \multicolumn{3}{|l|}{ Sítio tumoral } \\
\hline Tumor primário & 25 & 86,2 \\
\hline Tumor secundário & 4 & 13,8 \\
\hline Figado* & 2 & 50,0 \\
\hline Mama & 1 & 25,0 \\
\hline Colorretal & 1 & 25,0 \\
\hline \multicolumn{3}{|l|}{ Perfil histológico } \\
\hline Adenocarcinoma & 11 & 37,9 \\
\hline Escamocelular & 5 & 17,2 \\
\hline Carcinoide & 5 & 17,2 \\
\hline Metástase & 4 & 13,8 \\
\hline Outros tumores & 4 & 13,8 \\
\hline Carcinoma mисоерidermoide & 1 & \\
\hline Hamartoma pleuropulmonar & 1 & \\
\hline Hiperplasia adenomatosa atípica & 1 & \\
\hline Linfoma de zona marginal extranodal & 1 & \\
\hline \multicolumn{3}{|l|}{ Grau de diferenciação (CNPCs) } \\
\hline Bem diferenciado & 0 & 0 \\
\hline Moderadamente diferenciado & 12 & 75,0 \\
\hline Pouco diferenciado & 4 & 25,0 \\
\hline \multicolumn{3}{|l|}{ Expressão de PD-L1 (CNPCs) } \\
\hline Positivo & 2 & 12,0 \\
\hline Negativo & 14 & 88,0 \\
\hline \multicolumn{3}{|l|}{ Expressão de EGFR (CNPCs) } \\
\hline Mutado & 4 & 25,0 \\
\hline Não-mutado & 12 & 75,5 \\
\hline
\end{tabular}

Legendas: CNPCs: carcinoma não pequenas células. 
A análise comparativa entre o subtipo histológico e o sexo mostrou que, no sexo feminino, 07 casos foram de adenocarcinoma (36\%), 04 casos foram carcinoma de células escamosas (21\%), 04 casos de tumores carcinoide (21\%) e 02 casos de metástase de outros sítios (11\%), além de outros tumores de menor frequência. Nos pacientes do sexo masculino, 04 casos foram de adenocarcinoma (40\%), 01 caso de carcinoma de células escamosas $(10 \%), 01$ caso de tumor carcinoide (10\%), 02 casos de metástase de outros sítios $(20 \%)$, além dos demais tumores de menor frequência.

$\mathrm{Na}$ análise comparativa entre idade e tipos de carcinoma pulmonar, os casos de adenocarcinoma tiveram idade média de 58,9 $\pm 17,4$ (30-85 anos) e mediana de 66 anos; os casos de CEC tiveram idade média de 71,2 \pm 6,8 (62-80 anos) e mediana de 71 anos; os casos de tumor carcinoide tiveram idade média de 65,4 $\pm 11,3$ (50-82 anos) e mediana de 65 anos. Nos casos de metástase pulmonar, a idade média foi $69,8 \pm 9,0$
(59-81 anos) com mediana de 69,5 anos. Nos demais tipos de tumores pulmonares menos frequentes a idade média foi 43,3 $\pm 8,7$ (33-53 anos) e a mediana de 43,5 anos (Tabela 2).

Entre os adenocarcinomas, o grau de diferenciação mostrou que 7 eram moderadamente diferenciados, 3 eram bem diferenciados, e apenas 01 pouco diferenciado. Quanto ao subtipo histológico, 3 eram lepídicos, 5 eram acinares, 1 era papilífero e 2 eram sólidos.

A análise da expressão de PD-L1 e alterações EGFR nos CNPCs mostrou que 02 casos de adenocarcinoma expressaram PD-L1 (18\%). Todos os casos de CEC testados foram negativos. As alterações de EGFR foram positivas em 04 casos de adenocarcinoma (36\%). Todos os casos que apresentaram expressão de PDL-1 ou alterações EGFR foram de adenocarcinoma de grau histológico moderadamente diferenciado (Tabela 3).

Tabela 2. Análise comparativa entre a idade ao diagnóstico e sexo.

\begin{tabular}{lcccc}
\hline & $\begin{array}{c}\text { Total } \\
(\mathbf{n = 2 9 )}\end{array}$ & $\begin{array}{c}\text { Feminino } \\
(\mathbf{n = 1 9 )}\end{array}$ & $\begin{array}{c}\text { Masculino } \\
(\mathbf{n = 1 0 )}\end{array}$ & p-valor \\
\hline Média \pm Desvio Padrão (anos) & $61,5 \pm 15,2$ & $59,8 \pm 16,2$ & $64,7 \pm 13,2$ & \\
Mediana (anos) & 66 & 64 & 67 & 0,4168 \\
Mínimo (anos) & 30 & 30 & 45 & \\
Máximo (anos) & 85 & 82 & 85 & \\
\hline
\end{tabular}

Nota: ANOVA Summary.

Tabela 3. Análise da expressão de PD-L1 e alterações EGFR em carcinomas não pequenas células (CNPCs).

\begin{tabular}{lccccc}
\hline & \multicolumn{2}{c}{ Adenocarcinomas } & & CEC & p-valor \\
& $\mathbf{n}$ & $\mathbf{\%}$ & $\mathbf{n}$ & $\mathbf{\%}$ & \\
\hline $\begin{array}{l}\text { Expressão PD-L1 } \\
\text { Positivo }\end{array}$ & 2 & 18 & 0 & 0 & 1,000 \\
Negativo & 9 & 82 & 5 & 100 & \\
Alterações EGFR & & & & & \\
Mutado & 4 & 36 & 0 & 0 & 0,2445 \\
Não mutado & 7 & 64 & 5 & 100 & \\
\hline
\end{tabular}

Nota: ANOVA Summary.

Na análise comparativa da expressão de PD-L1 e tipos histológicos de adenocarcinomas, foi observado que 01 caso era tipo acinar (50\%) e 01 caso tipo lepídico (50\%). Todos os adenocarcinomas que apresentaram alterações EGFR eram do tipo acinar. A mutação EGFR encontrada em todos os pacientes foi posição C01:C03, mutação L858R no éxon 21 (Gráfico 1).

Do total de tumores carcinoides, 04 casos (80\%) foram carcinoides típicos e 01 caso $(20 \%)$ foi de carcinoide atípico.
Todos os casos de carcinoide típico ocorreram em pacientes do sexo feminino, que apresentaram idade média de $65,5 \pm$ 13,1 anos (50-82 anos) e mediana de 65 anos. O único caso analisado de carcinoide atípico foi em um paciente do sexo masculino de 65 anos de idade.

O perfil imuno-histoquímico dos casos de adenocarcinoma mostrou que todos apresentaram imunoexpressão de TTF1 e ausência de marcação para p63, dentre outros marcadores realizados. O perfil imuno-histoquímico dos casos de 
carcinoma de células escamosas mostrou o contrário. Todos os casos apresentaram negatividade para o marcador TTF-1 e imunoexpressão para p63. Nestes casos também houve imunoexpressão marcante de ck5/6 e 34BE12 em elevado percentual dos casos. No painel de imuno-histoquímica dos casos de tumores carcinoides foi observado predomínio de marcação em todos os casos de Cromogranina A e Sinaptofisina, sendo o ki67 baixo nos casos de carcinoide típico, cerca de 2 a 3\%, e elevados nos casos de carcinoide atípico com positividade de até $40 \%$.

Gráfico 1. Análise comparativa entre subtipo histológico de adenocarcinoma e expressão de PD-L1.

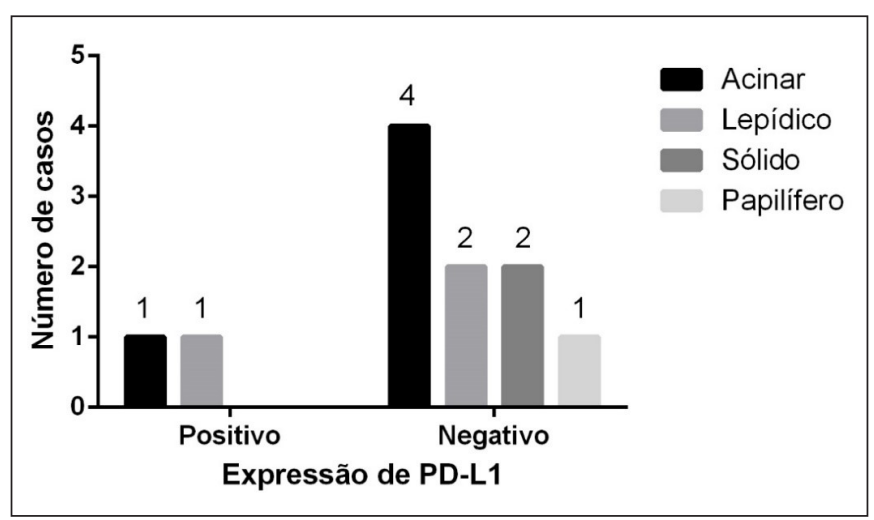

\section{DISCUSSÃO}

O câncer de pulmão é mais prevalente no sexo masculino, na idade de 60 anos ou mais, porém, vem sendo observada uma tendência crescente na mortalidade entre as mulheres nas últimas décadas, pois observa-se o aumento do tabagismo entre as mulheres. ${ }^{12}$ Neste atual estudo, evidenciou-se uma maior prevalência no sexo feminino, respondendo por $66 \%$ do total de casos analisados. A idade média encontrada foi de 61 anos com mediana de 66 anos. Este estudo revelou ainda que a idade média em homens foi levemente superior que a idade em mulheres, 64 anos em comparação com 59 anos, respectivamente, estando de acordo com a epidemiologia desta doença. ${ }^{13}$

A maioria dos casos deste estudo mostrou tratar-se de tumores primários, 25 casos $(86 \%)$, contra apenas 04 casos (14\%) de tumores secundários, sendo 02 casos de metástase de hepatocarcinomas, 01 de carcinoma mamário e 01 de adenocarcinoma colorretal.

A metástase pulmonar pode ocorrer em portadores de neoplasias extratorácicas e a incidência varia de $20 \%$ a $54 \%$ nos pacientes acometidos por câncer. Os sítios primários que mais dão metástase para o pulmão são mama, cólon, rim, útero e carcinomas da região cabeça e pescoço. ${ }^{14}$

Um dado interessante deste estudo é que todos os casos de metástase de hepatocarcinoma foram pós-transplante hepático; dado que está de acordo com estudos prévios que mostram que até $56 \%$ dos pacientes pós-transplante de fígado podem apresentar lesões secundárias pulmonares, onde o retorno venoso que flui pro pulmão através do sistema vascular geralmente acomete o pulmão com as metástases extratorácicas. $^{15}$

O tipo histológico predominante entre os tumores primários do pulmão é adenocarcinoma, seguido pelos CEC. Este trabalho mostrou um percentual de $38 \%$ de adenocarcinomas e $17 \%$ de CEC. O tipo mais comum de adenocarcinoma é o acinar, seguido dos tipos sólido, micropapilar, papilar e lepídico. ${ }^{16}$ Observou-se neste estudo que dos 11 casos de adenocarcinoma, 05 foram tipo acinar, 03 tipo lepídico, 02 tipo sólido e 01 tipo papilífero.

Alguns estudos mostram que o adenocarcinoma seria mais prevalente em mulheres, já em homens, o carcinoma de células escamosas seria o mais comum. ${ }^{17}$ Este atual estudo mostrou que no sexo feminino houve 07 casos de adenocarcinomas, contra 04 casos do sexo masculino.

A idade média de apresentação dos tipos de carcinomas pulmonares também é distinta segundo alguns estudos, sendo os adenocarcinomas mais comuns em idade média superior a 55 anos, o carcinoma de células escamosas com idade média superior a 60 anos e os carcinoides com idade média entre 55 e 60 anos. ${ }^{17-20}$ Este trabalho revelou que a idade média encontrada foi de 58 anos para adenocarcinomas, 71 anos para CEC e 65 anos para carcinoide.

A expressão de PDL-1 é mais comumente observada nos adenocarcinomas moderadamente e pouco diferenciados, com estudos revelando ainda maior percentual de expressão em tipo sólido, em sexo masculino e na faixa etária entre 50 e 70 anos. ${ }^{8,10,21}$ Este trabalho mostrou que do total de casos analisados de adenocarcinoma, apenas 02 foram positivos e 09 foram negativos. Já em CECs este estudo não revelou imunoexpressão de PD-L1.

As alterações de EGFR são mais comumente observadas nos adenocarcinomas do tipo acinar com maior percentual no sexo feminino e na idade inferior ou igual a 70 anos. ${ }^{8}$ Este estudo mostrou que 04 casos (36\%) apresentavam alterações EGFR, sendo todos adenocarcinomas acinares, sendo 3 no sexo feminino e 4 no sexo masculino.

Estudos revelam ainda que a expressão de PDL-1 pode estar relacionada ou não a alterações de EGFR, sendo esta associação mais observada no adenocarcinoma tipo acinar, principalmente em pacientes mais velhos com idade superior a 70 anos e do sexo masculino..$^{8,22-24}$ Este estudo não mostrou relação entre os casos que apresentaram imunoexpressão de PD-L1 e alterações EGFR.

O local mais comum de ocorrência de alterações no EGFR é uma deleção no éxon 19 e a mutação L858R no éxon $21 .^{24,25}$ Neste estudo todos os pacientes apresentavam mutações na posição C01:C03, e mutação L858R no éxon 21.

A literatura mostra que os tumores carcinoides mais prevalentes são típicos no sexo feminino e com idade média entre 55 e 60 
anos. ${ }^{26}$ Este trabalho revelou que $80 \%$ dos casos foram típicos e $20 \%$ atípicos, sendo mais comuns em mulheres com idade média de 65 anos.

A recomendação padrão para análises de tumores é que se façam os marcadores essenciais a fim de não haver desgaste do material coletado na biópsia, sendo recomendada pesquisa inicial com TTF1 e p63 ou p40, a fim de elucidação diagnóstica e, somente após isso, poder-se-á realizar extensão do painel, caso seja necessário. ${ }^{3}$ Em adenocarcinomas, a grande maioria é positiva para TTF1 e negativa para p63. Os CECs são em grande maioria negativos para TTF1 e positivos para $\mathrm{p} 63 .{ }^{27}$ Já os tumores carcinoides costumam expressar cromogranina, sinaptofisina, CD56 e demais marcadores neuroendócrinos, além de costumarem apresentar índice de proliferação celular pelo ki67 $\leq 5 \%{ }^{3,28}$

Neste estudo revelou-se expressão para TTF-1 e ausência de marcação para p63 em todos os casos de adenocarcinomas. Os casos de CECs mostraram o oposto, onde todos os casos apresentaram negatividade para o marcador TTF-1 e para a expressão para p63. Os casos de carcinoides revelaram marcação dos reagentes neuroendócrinos: cromogranina e sinaptofisina, sendo o ki67 baixo em carcinoides típicos, cerca de 2 a $3 \%$, e elevado no caso do paciente com tumor carcinoide atípico, com positividade de até $40 \%$.

Outros tumores encontrados neste estudo foram CME de baixo grau endobrônquico, hamartoma pleuropulmonar, hiperplasia adenomatosa atípica e linfoma de zona marginal extranodal. O CME é um carcinoma tipo glândula salivar símile que corresponde de $0,3 \%$ a $1 \%$ de todas as neoplasias malignas e tende a apresentar crescimento lento. A literatura médica mundial mostra que os hamartomas são tumores benignos raros e mais comuns em adultos. Linfomas de zona marginal extranodal são os linfomas mais comuns entre os tipos primários pulmonares, tendo progressão lenta e sendo curáveis em estágios iniciais. . $^{1,29,30}$

\section{CONCLUSÕES}

O presente trabalho mostrou a relevância do estudo imuno-histoquímico e da realização de PCR para pesquisa de alterações EGFR na avaliação de fatores prognósticos, identificação dos diferentes tipos histológicos, definição de casos primários e determinação de sítios primários dos tumores secundários, a fim de contribuir no direcionamento do tratamento dessa doença e melhorar a qualidade de vida destes pacientes.

A pesquisa de alterações de EGFR pela técnica de PCR se mostra primordial, pois com o tratamento adequado há um impacto no prognóstico dos pacientes. Os resultados coletados corroboram, em sua maioria, com dados já descritos na literatura.

A maior prevalência de casos em mulheres contrasta com os dados da literatura, mas estudos já mostram que há uma tendência crescente de casos de câncer de pulmão entre as mulheres. Os traços são semelhantes a perfil já conhecido de pacientes com câncer de pulmão, como a positividade de TTF-1 e negatividade para p63, e o adenocarcinoma sendo o tipo histológico predominante. Os casos de metástase de hepatocarcinoma pós-transplante concordam com pesquisas prévias que mostram a prevalência de lesões secundárias pulmonares nesses pacientes transplantados.

\section{REFERÊNCIAS}

1. Brasil. Ministério da Saúde. Instituto Nacional de Câncer (INCA) [Internet]. Câncer de pulmão. Brasília (DF): Ministério da Saúde; 2019. [Acesso em: 20 de novembro de 2020]. Disponível em: https:// www.inca.gov.br/tipos-de-cancer/cancer-de-pulmao

2. Santos GC, Shepherd FA, Tsao MS. EGFR Mutations and Lung Cancer. Annual Reviews of Pathology: mechanisms of disease. Annu Rev Pathol . 2011;6:49-69.

3. Capellozi VL. Role of immunohistochemistry in the diagnosis of lung cancer. J Bras Pneumol . 2009;35(4):375-82.

4. American Cancer Society. What Is Lung Cancer? [Internet] American Cancer Society; 2019. [Acesso em: 20 de novembro de 2020]. Disponível em: https://www.cancer.org/cancer/lung-cancer/ about/what-is.html

5. Herbst RS. Review of epidermal growth factor receptor biology. Int J Radiat Oncol Biol Phys . 2004;59(2 Suppl):21-6.

6. Lopes GL, Vattimo EFQ, Castro Junior G. Identifying activating mutations in the EGFR gene: prognostic and therapeutic implications in non-small cell lung câncer. Jornal Brasileiro de pneumologia. 2015;41(4):365-75.
7. Ilie M, Hofman V, Dietel M, Soria JC, Hofman O. Assessment of the PD-L1 status by immunohistochemistry: challenges and perspectives for therapeutic strategies in lung cancer patients. Virchows Arch. 2016;468(5):511-25.

8. Oliveira AC, Silva AV, Alves M, Cronemberger E, Carneiro BA, Melo JC, et al. Perfil molecular do carcinoma pulmonar de células não pequenas no Nordeste brasileiro. J Bras Pneumol. 2019;45(3):e20180181.

9. Rittmeyer A, Barlesia F, Waterkamp D, Park K, Ciardiello F, von Pawel J, et al. Atezolizumab versus docetaxel in patients with previously treated non-small-cell lung cancer (OAK): a phase 3, open-label, multicentre randomised controlled trial. Lancet. 2017;389(10066):255-65.

10. Lima KA. Análise de expressão do PD-L1 em pacientes com câncer de pulmão de não pequenas células na região sul do Brasil [Internet]. Florianópolis: UFSC; 2019. [Acesso em: 20 de novembro de 2020]. Disponível em: http://repositorio.ufsc.br/ handle/123456789/204044

11. Pardoll DM. The blockade of imune checkpoints in câncer immunotherapy. Nat Rev Cancer. 2012;12(4):252-64. 
12. Silva GA, Pompeiano NC, Oliveira SM, Pinto OJ. Diferenças de gênero na tendência de mortalidade por câncer de pulmão nas macrorregiões brasileiras. Rev Bras Epidemiol. 2008;11(3):411-9.

13. Uehara C, Jamnik S, Santoro IL. Câncer de pulmão. Medicina (Ribeirão Preto). 1998;31(2):266-76.

14. Hirakata K, Nakata H, Nakagawa T. CT of pulmonary metastases with pathological correlation. Semin Ultrasound CT MR. 1995;16(5):379-94.

15. Schlitt HJ, Neipp M, Weimann A, Oldhafer KJ, Schmoll E, Boeker $\mathrm{K}$, et al. Recurrence patterns of hepatocellular and fibrolamellar carcinoma after liver transplantation. J Clin Oncol. 1999;17(1):324-31.

16. The Cancer Genome Atlas Research Network. The Cancer Genome Atlas Research Network. Comprehensive molecular profiling of lung adenocarcinoma. Nature. 2014;511(7511):543-50.

17. Tsukazan MTR, Vigo A, Silva VD, Barrios CH, Rios JO, Pinto JAF. Câncer de pulmão: mudanças na histologia, sexo e idade nos últimos 30 anos no Brasil. J Bras Pneumol. 2017;43(5):363-7.

18. Colaço MI, Estrada H, Moreno R, Veloso B. Carcinoma do pulmão. Revisão da Casuística de 12 Anos num Serviço de Medicina Interna. Acta Med Port. 1992;5(7):365-8.

19. Sagerup CMT, Smastuen M, Johannesen TB, Helland A, Brustugun OT. Increasing age and carcinoma not otherwise specified: a 20-year population study of 40,118 lung cancer patients. Lung Cancer Patients. J Thorac Oncol. 2012;7(1):57-63.

20. Hobbins S, West D, Peake M, Beckett P, Woolhouse I. Patient characteristics, treatment and survival in pulmonary carcinoid tumours: an analysis from the UK National Lung Cancer Audit. BMJ Open. 2016;6(9):e012530.

21. Alexandre IA. Estudo da expressão imuno-histoquímica do PDL1 em carcinoma de pulmão não-pequenas células: correlação com dados clínicos patológicos [Internet]. Florianópolis: Repositório Institucional UFFS; 2018. [Acesso em: 20 de novembro de 2020]. Disponível em: https://rd.uffs.edu.br/handle/prefix/3106
22. Takada K, Toyokawa G, Tagawa T, Kohashi K, Shimokawa M, Akamine T, et al. PD-L1 expression according to the EGFR status in primary lung adenocarcinoma. Lung Cancer. 2018;6:1-6.

23. Yang H, Chen H, Luo S, Li L, Zhou S, Shen R, et al. The correlation between programmed death-ligand 1 expression and driver gene mutations in NSCLC. Oncotarget. 2017;8(14):2351728.

24. Melo AC, de Sa VK, Sternberg C, Olivieri ER, da Cunha IW, Fabro AT, et al. Mutational profile and new IASLC/ATS/ERS classification provide additional prognostic information about lung adenocarcinoma: a study of 125 patients from Brazil. Oncology. 2015;89(3):175-86

25. Inoue A, Yoshida K, Morita S, Imamura F, Seto T, Okamoto I, et al. Characteristics and overall survival of EGFR mutation-positive non-small cell lung cancer treated with EGFR tyrosine kinase inhibitors: a retrospective analysis for 1660 Japanese patients. Jpn J Clin Oncol. 2016;46(5):462-7.

26. Mccaughan BC, Martini N, Bains MS. Bronchial carcinoids: review of 124 cases. J Thorac Cardiovasc Surg. 1985;89(1):8-17.

27. Kargi A, Gurel D, Tuna B. The diagnostic value of TTF-1, CK $5 / 6$, and p63 immunostaining in classification of lung carcinomas. Appl Immunohistochem Mol Morphol . 2007;15(4):415-20.

28. Gross JL, Vega MA, Frenhi GS, Torres SM, Campos AH, Pinto CA, et al. Bronchial carcinoid tumors: second primary neoplasms and outcomes of surgical treatment. J bras pneumol. 2019;45(5):e20180140.

29. Wang ZZ, Li D, Li J, Gao Y, Zhang XF, Liu S. [Clinical analysis of 5 cases of endobronchial hamartoma]. Zhonghua Jie He He Hu Xi Za Zhi. 2019;42(1):15-19.

30. A.C.Camargo Cancer Center. Linfoma não Hodgkin [Internet]. São Paulo; 2019. [Acesso em: 20 de novembro de 2020]. Disponível em: https://www.accamargo.org.br/sobre-o-cancer/tipos-de-cancer/ linfoma-nao-hodgkin

\section{Como citar:}

Soares CE, Xavier PM, Oliveira AC, Gerson G. Utilização das técnicas de imuno-histoquímica e reação em cadeia da polimerase (PCR) no diagnóstico, na avaliação de fatores prognósticos e no direcionamento do tratamento do câncer de pulmão. Rev Med UFC. 2021;61(1):1-7. 